\title{
Aerial transmission of the SARS-CoV-2 virus through environmental e-cigarette aerosol: is it plausible?
}

\author{
Roberto Sussman ${ }^{1}$, Eliana Golberstein ${ }^{2}$, Riccardo POLOSA $^{3}$ \\ 1 Universidad Nacional Autónoma de México \\ 2 Myriad RBM \\ 3 University of Catania
}

Funding: No external funding was received.

Potential competing interests: R.A.S. declares no competing interests. E.G is currently employed by Myriad Pharmaceuticals, an independent company that manufactures e-liquids and vaping devices in New Zealand. She also provides consultancy work on research and development, regulatory affairs support, and formulation to several independent vaping companies in the Pacific Region. In the past she has worked for several pharmaceutical companies, including GlaxoSmithKline and Genomma Lab. She is also a member of the standards committee of the VTANZ and UKVIA. R.P. is a full-time employee of the University of Catania, Italy. RP is Medical Director of the Institute for Internal Medicine and Clinical Immunology at the Universiy of Catania, Italy. In relation to his work in the area of tobacco control and respiratory diseases, RP has received lecture fees and research funding from Pfizer, GlaxoSmithKline, CV Therapeutics, NeuroSearch A/S, Sandoz, MSD, Boehringer Ingelheim, Novartis, Duska Therapeutics, and Forest Laboratories. He has also served as a consultant for Pfizer, Global Health Alliance for treatment of tobacco dependence, CV Therapeutics, NeuroSearch A/S, Boehringer Ingelheim, Duska Therapeutics, Forest Laboratories, ECITA (Electronic Cigarette Industry Trade Association, in the UK), and Health Diplomats. RP has served on the Medical and Scientific Advisory Board (MSAB) of Cordex Pharma, Inc., CV Therapeutics, Duska Therapeutics Inc, Pfizer, and PharmaCielo. Lecture fees from a number of European EC industry and trade associations (including FIVAPE in France and FIESEL in Italy) were directly donated to vaper advocacy no-profit organizations. RP is also founder of: 1) the Center for Tobacco prevention and treatment (CPCT) at the University of Catania; and 2) the Center of Excellence for the acceleration of Harm Reduction (CoEHAR) at the same University, which has received support from Foundation for a Smoke Free World to conduct 8 independent investigator-initiated research projects on harm reduction. He is currently scientific advisor for LIAF, Lega Italiana Anti Fumo (Italian acronym for Italian Anti-Smoking League) and Head of the European Technical Committee for standardization on "Requirements and test methods for emissions of electronic cigarettes" (CEN/TC 437; WG4).

\section{Abstract}

We discuss the plausibility, scope and risk for SARS-CoV-2 virus transmission through respiratory droplets potentially carried by e-cigarette aerosol (ECA) exhaled by infected vapers. Considering observational data on droplet emission rates of mouth breathing as a proxy model for this transmission, droplet diameters should be overwhelmingly in the submicron range. For the most common low intensity puffing style (practiced by $80-90 \%$ of vapers) we estimate emission rates of 2-230 droplets per puff horizontally transported 0.5-2 meters in the direction of the exhaled jet. Considering that vaping is an intermittent respiratory expiration associated with a short duration exposure and assuming contagion risks to be proportional to the SARS-CoV-2 viral load of emitted droplets, we estimate that vaping represents about $1 \%$ added risk with respect to the ever existing risk from continuous rest breathing in indoor spaces with natural ventilation (as a reference, speaking for 6 minutes per hour increases this risk to 44\%). The added risks remain negligible with universal wearing of face masks protecting bystanders.

Direct aerial transmission of the SARS-CoV-2 virus borne by respiratory droplets is a fact already acknowledged by the WHO [1] and the CDC [2]. Indirect transmission by smaller droplets (often referred to as 'aerosols') is also proven [3], but its reach and frequency remain controversial $[4,5]$. The current COVID19 pandemic has intensified the existing scientific 
interest in the mechanisms of generation, viral transport and respiratory droplet dynamics emitted by different respiratory activities, such as respiration [6], vocalization [7], coughing [8] and sneezing [9]. In this paper we expand this current body of study by addressing the plausibility of SARS-CoV-2 virus transmission via respiratory droplets borne by e-cigarette aerosol (ECA) exhaled by infected vapers. For a more detailed presentation of the discussion given here, see [10].

Vaping is characterized by a wide variety of individual styles or topographies that have a range of intensities depending on whether the devices are low or high-powered [11,12,13]. However, we address here the 'mouth-to-lung '(MTL) style, requiring momentary mouth retention of the aerosol bolus prior to lung inhalation, which involves low powered devices and is practiced by the overwhelming majority (80-90\%) of vapers [14] (see [10] for a discussion of droplet emission in minority styles and topographies).

Droplets exhaled by an infected vaper would not be just "airborne" as in other respiratory expirations, but would be evolving jointly with a non-biological aerosol (ECA) that is strongly diluted in exhaled air. Hence, considering inhaled ECA droplet numbers [15] and a 90\% retention by the respiratory system [16,17], the exhaled respiratory droplets would be accompanying a much larger number (about $10^{8}-10^{9}$ ) of submicron ECA droplets composed of propylene glycol (PG), vegetable glycerine (VG), nicotine and water [18].

As there is no experimental data on respiratory droplets borne by ECA, we need to infer their diameter distributions and emission rates by looking at existing evidence on expiratory behaviors that can serve as proxies for vaping. Therefore, we first need to measure the exhaled quantity of air diluted ECA that vapers exhale. Cigarette smoking is a helpful proxy for this purpose, as most vapers are either relatively recent ex-smokers or current smokers, often following the style of MTL vaping that resembles the topography of puffing practiced by most cigarette smokers $[19,20]$. Available evidence $[21,22]$ indicates that smokers exhale a fluid mixture of mainstream smoke and air with a total volume $30-40 \%$ greater than the normal tidal resting breathing volume (approximately $700-900$ vs. $400-600 \mathrm{~cm}^{3}$ ). This rise in volume is the probable product of smoke suction and is also consistent with smoking occupying a higher percentage of vital capacity (approximately $20 \%$ versus $10-15 \%$ in rest breathing [21]).

We conclude that is fair to assume this quantity of exhaled fluid volume for vaping, an inference further justified by the fact that it requires higher air flow resistance than smoking [23], though vapers puff for longer periods [11,12,13], thus being able to inhale a comparable (or greater) aerosol mass and puff volume (between 4-10 mg and $20-100 \mathrm{~cm}^{3}[11,12,13]$ ) as smokers by exerting similar effort (similar suction pressure gradient).

We consider mouth breathing as a useful proxy model to infer respiratory droplet diameters and emission rates by vaping, given the lack of empirical evidence. To explain this, we estimate vaping-related exhalation velocities. A total volume of fluid of 300-1500 cm $\mathrm{cm}^{3}$ exhaled in 2-4 seconds through the combined nose/mouth area of 2-3 $\mathrm{cm}^{2}$ (vaping does not include nose occlusion) induces exhalation speeds of $U_{0}=30-250 \mathrm{~cm} / \mathrm{s}$. The range of these exhalation velocities is comparable to that of measured velocities for mouth breathing [24,25], which are below velocities for speaking $(3.9 \mathrm{~m} / \mathrm{s})$, coughing (6$22 \mathrm{~m} / \mathrm{s})$ and sneezing $(20-35 \mathrm{~m} / \mathrm{s})$ reported in the literature $[26,27,28,29,30]$. Hence, we can consider the wealth of 
available data on respiratory droplets from breathing experiments at different levels of inspiration (see review in [6]), with subjects breathing in all cases through mouthpieces and noseclips.

Mouthpieces and noseclips are standard instruments in breathing experiments. This is not necessarily a drawback since vaping involves oral inhalation by suction through a mouthpiece (but not nose occlusion that occurs by wearing noseclips). Thus, we examined the available literature $[31,32,33]$ that looks at the effects of these instruments in breathing parameters. While oral inhalation with a mouthpiece and a noseclip increases tidal volume and inhalation/exhalation times for factors close to $20 \%$ above rest breathing values, these effects practically vanish when nasal breathing is not occluded [33], even if subjects are kept breathing orally through a mouthpiece, as is the case in vaping (and smoking). This would indicate a volume of exhaled fluid similar to the volume of rest breathing tidal volume $\left(400-600 \mathrm{~cm}^{3}\right)$ for vaping, but the studies examining the effects of mouthpieces and noseclips did not consider aerosol suction that occurs in vaping. Therefore, our estimate of exhaled fluid volume due to vaping is approximately comparable to that showing a rise of exhaled fluid volume in studies using mouthpieces and noseclips on respiratory droplets from mouth breathing.

Therefore, experimental breathing data (see analysis in $[6,10]$ ) can be used as a reliable proxy to estimate droplet emissions for vaping, leading to an estimated emission rate of between 2 and 230 droplets per puff, overwhelmingly in the submicron range. This rate of emission is comparable to droplet numbers and sizes observed in respiratory experiments for tidal volumes close to rest breathing.

Since both ECA and respiratory droplets are in the submicron range, they exert negligible influence on the flow dynamics of the carrier fluid. In other words: exhaled ECA is a "single phased" fluid flow [34] in which ECA droplets act approximately as molecular contaminants that can serve as tracers to visualize the respiratory air flow (because of their optical properties [35]). In fact, usage of tracer gases and aerosols with fine and hyper fine particles like ECA is a standard technique to visualize respiratory flows [36].

After the exhaled tidal volume and numbers and diameters of respiratory droplets to be borne by the exhaled ECA have been inferred, we continue to estimate the direct exposure distance. We model ECA flow as a puff with a starting turbulent jet with finite fluid injection (finite exhalation time) $[37,38,39]$ to estimate how far respiratory droplets can be carried by exhaled ECA. The dynamical model yields a range of 0.5-2.0 meters for horizontal transported of (ECA and respiratory) droplets (see detailed discussion in [10]). Once the injection ceases, the jet transforms into an unstable puff that is rapidly disrupted by induced air and turbulent mixing [37], with submicron droplets (ECA and respiratory) transported by the jet subsequently dispersed by air currents, raising a potential risk of indirect contagion.

We consider (see [10]) a condensed version of the dose-response exponential risk model proposed by Buonanno, Morawska and Stabile (BMS) to evaluate the risk of indirect contagion $[40,41]$. Our goal is to estimate the relative risk of indoor vaping, an intermittent expiratory operation, with respect to (and in direct contrast with) rest breathing, which is an inevitable continuous expiratory activity that can be regarded as a " control case " scenario. For speaking and coughing, 
which are often episodic and sporadic behaviors, the same risk relation with respect to this " control case " can be calculated.

Assuming that the submicron respiratory droplets have been spread uniformly and considering recent data used by BMS on SARS-CoV-2 viral load and other infection parameters, as well as their data on droplet size and emission rates, we evaluate these relative risks for a home and restaurant scenarios (12 and 3 hours total exposure) with natural and mechanical ventilation. The resulting values of added risks with respect to the control case are:

- $1 \%$ for vaping (160 daily puffs)

- $44 \%$ for continuous speaking $10 \%$ of time (6 minutes every hour), up to $90 \%$ for speaking $40 \%$ of time (20 minutes every hour)

- over $260 \%$ for coughing 30 times per hour.

Last but not least, we comment on the possibility of chemical interaction between respiratory droplets and PG and VG vapors, the main chemical compounds of the gas phase of ECA. These compounds are glycols whose bactericidal and virucidal properties $[42,43]$ and aerial disinfectant effects have been laboratory tested on various pathogens (mostly bacteria) $[44,45,46]$. However, the highly idealized conditions and PG concentrations under which these disinfectant experiments were conducted could hardly be applicable to the erratic and intermittent conditions of realistic vaping. Nevertheless, it should be worthwhile and useful to test experimentally the disinfectant properties of PG on the SARSCoV-2 virus.

The study we have undertaken has obvious limitations. We inferred estimates for droplet emission through ECA by extrapolating from available data on droplet sizes and emission rates of cigarette smoking and mouth breathing taken as proxies. While these estimates are consistent with the physical and chemical properties of ECA, and/or the relation between vaping and these proxies, they still need to be corroborated by actual experimental and observational studies.

We accept that our fluid dynamic modeling of exhaled ECA is idealized, but we claim that the calculation of direct exposure distances from a single vaper in still air under natural ventilation is acceptable and necessary. Advanced theoretical methods of fluid mechanics (see for example [47]) would be sufficient to estimate indirect exposure by studying droplet dynamics under more realistic conditions (a ventilation regime and integrating the effects of turbulent air mixing and thermal convection). Rather, we examined indirect exposure through a simplified exponential risk model based on the rates of expired viral load through various respiratory activities of actual SARS-CoV-2 data. This simplified risk model is also idealized, but it fulfills our aim of providing a rough estimation of relative risks from indirect exposure to vaping (as an intermittent expiration) with respect to the control case of continuous rest breathing. We believe that this simplified approach captures the essential risk information to guide prevention policies, though a more elaborate and complete approach should be attempted in a future analysis (see [10] and references cited therein). 
As commented before, an interesting feature resulting from a possible respiratory droplet transport by vaping is the possibility of actually visualizing a potentially risky respiratory airflow. This has an important psychological dimension that is absent in other expiratory activities that cannot be visualized (speaking, singing, coughing, sneezing) but are also potentially carrying infective droplets. Thus, bystanders able to visualize ECA as a respiratory flow would be able to position themselves at appropriate safe distances for direct exposure (1-2 meters), approximately equal to the recommended distances of social separation. Respiratory flow visualization would also make it clear that the distances for direct exposure risk applies only in the direction of the exhaled jet, with only indirect exposure risk to individuals positioned in other directions (and not wearing face masks). Nevertheless, if you do not wear a face mask, it is prudent to maintain 2 meters of separation from anyone vaping.

In indoor or socially shared spaces where there is no universal wearing of face masks, vaping will add only a minuscule additional risk (1\%) to those risks already existing from continuous breathing or talking. Face masks worn by infected persons offer fairly effective protection against contamination by infected persons, but wearing them also provides bystanders reasonably good protection from emissions from people infected who are not wearing a face mask [48].

Evidently, universal wearing of face masks implies a drastic reduction of the existing baseline risk for continuous breathing (more so for talking) in every place. It can be argued that vaping involves momentary face mask removal and thus would increase exposure risks in an indoor environment where everyone is wearing a face mask. However, vaping is not the only activity that would involve such an increase of risk: eating and drinking are impossible without fully removing face masks, whereas it is possible to vape by momentarily adjustment (not necessarily full removal) of a face mask. Nevertheless, if face mask wearing is universal this risk enhancement would be negligible and inconsequential because the same face masks would protect those wearing them from short intermittent emissions by others when eating or drinking (and including those from the vaper).

Breathing emissions due to brief intermittent removal or adjustment of a face mask to drink, eat or vape, or even to take a brief rest from wearing the mask, would imply for bystanders (already protected for wearing face masks) to tolerate a small rate of droplet emission only for a very short time. Since face mask cannot be rigidly maintained and enforced $100 \%$ of time in shared spaces, this tolerance is necessary for civilized coexistence. In the specific case of vaping it implies a tolerance of mask-free periods that would be of shorter duration than those for eating or drinking: likely no longer than 10 seconds roughly 10-15 times per hour (being free from this exposure for the remaining 600-1400 breaths by the vaper in the same hour).

It is true that vaping might introduce risks not related to aerial transmission, such as from face touching, or mask manipulation or sharing or manipulating a device that will be placed in the mouth, but the same risks are present (and are tolerated or addressed by hygiene prevention) when drinking or eating. The same tolerance and courtesy given for people drinking or eating can (and should) be extended to vapers, most of whom are trying to stop smoking and stay smoke-free (or at least to smoke less). 
The risk for direct and indirect COVID-19 contagion from indoor vaping expirations does exist and must be taken into consideration. However, this risk must be placed in its proper context with respect to the parameters of exposure that characterize vaping and other expiratory activities. Therefore, as far as protection against SARS-CoV-2 virus is concerned, vaping in a home scenario or in shared social spaces does not require special extra interventions besides those already recommended for the general population: social distance and wearing face masks. Vapers should be advised to be alert to the worries and fears of non-vapers when sharing indoor spaces or dwellings or when close to other citizens, and for safety measures to use low-powered devices for low intensity vaping. Vapers, however, deserve the same sensitivity, courtesy and tolerance as well.

\section{Competing interests}

R.A.S. declares no competing interests.

E.G is currently employed by Myriad Pharmaceuticals, an independent company that manufactures e-liquids and vaping devices in New Zealand. She also provides consultancy work on research and development, regulatory affairs support, and formulation to several independent vaping companies in the Pacific Region. In the past she has worked for several pharmaceutical companies, including GlaxoSmithKline and Genomma Lab. She is also a member of the standards committee of the VTANZ and UKVIA.

R.P. is a full-time employee of the University of Catania, Italy. RP is Medical Director of the Institute for Internal Medicine and Clinical Immunology at the Universiy of Catania, Italy. In relation to his work in the area of tobacco control and respiratory diseases, RP has received lecture fees and research funding from Pfizer, GlaxoSmithKline, CV Therapeutics, NeuroSearch A/S, Sandoz, MSD, Boehringer Ingelheim, Novartis, Duska Therapeutics, and Forest Laboratories. He has also served as a consultant for Pfizer, Global Health Alliance for treatment of tobacco dependence, CV Therapeutics, NeuroSearch A/S, Boehringer Ingelheim, Duska Therapeutics, Forest Laboratories, ECITA (Electronic Cigarette Industry Trade Association, in the UK), and Health Diplomats. RP has served on the Medical and Scientific Advisory Board (MSAB) of Cordex Pharma, Inc., CV Therapeutics, Duska Therapeutics Inc, Pfizer, and PharmaCielo. Lecture fees from a number of European EC industry and trade associations (including FIVAPE in France and FIESEL in Italy) were directly donated to vaper advocacy no-profit organizations. RP is also founder of: 1) the Center for Tobacco prevention and treatment (CPCT) at the University of Catania; and 2) the Center of Excellence for the acceleration of Harm Reduction (CoEHAR) at the same University, which has received support from Foundation for a Smoke Free World to conduct 8 independent investigator-initiated research projects on harm reduction. He is currently scientific advisor for LIAF, Lega Italiana Anti Fumo (Italian acronym for Italian Anti-Smoking League) and Head of the European Technical Committee for standardization on "Requirements and test methods for emissions of electronic cigarettes" (CEN/TC 437; WG4). 


\section{References}

[1] W. H. O. Transmission of SARS-CoV-2: implications for infection prevention precautions: scientific brief, 09 july 2020 , Tech. rep., World Health Organization (2020). https://www.who.int/publications/i/item/modes-of-transmission-of-viruscausing-covid-19-implications-for-ipc-precaution-recommendations

[2] S. Brief, SARS-CoV-2 and potential airborne transmission. CDC org (2020). https://www.cdc.gov/coronavirus/2019ncov/more/scientific-brief-sars-cov-2.html

[3] Y. Liu, Z. Ning, Y. Chen, M. Guo, Y. Liu, N. K. Gali, L. Sun, Y. Duan, J. Cai, D. Westerdahl, et al., Aerodynamic analysis of sars-cov-2 in two Wuhan hospitals, Nature 582 (7813) (2020)

$557-560$

[4] L. Morawska, J. Cao, Airborne transmission of sars-cov-2: The world should face the reality, Environment International (2020) 105730

[5] National Academies of Sciences, Engineering, and Medicine, Airborne Transmission of SARS-CoV-2: Proceedings of a Workshop in Brief, The National Academies Press, Washington, DC, 2020. doi:10.17226/25958

[6] B. Bake, P. Larsson, G. Ljungkvist, E. Ljungström, A. Olin, Exhaled particles and small airways, Respiratory research 20 (1) (2019) 8

[7] S. Asadi, A. S. Wexler, C. D. Cappa, S. Barreda, N. M. Bouvier, W. D. Ristenpart, Aerosol emission and superemission during human speech increase with voice loudness, Scientific reports 9 (1) (2019) 1-10

[8] Zayas, G., Chiang, M.C., Wong, E. et al. Cough aerosol in healthy participants: fundamental knowledge to optimize droplet-spread infectious respiratory disease management. BMC Pulm Med 12, 11 (2012)

[9] Han, Z. Y., Weng, W. G., \& Huang, Q. Y. (2013). Characterizations of particle size distribution of the droplets exhaled by sneeze. Journal of the Royal Society Interface, 10(88), 20130560.

[10] Sussman, R. A. Golberstein, E. and Polosa, R. Aerial transmission of SARS-CoV-2 virus (and pathogens in general) through environmental e-cigarette aerosol. medRxiv 2020.11.21.20235283;

doi: https://doi.org/10.1101/2020.11.21.20235283

[11] B. Dautzenberg, D. Bricard, Real-time characterization of e-cigarettes use: the 1 million puffs study, J. Addict. Res. 
Ther 6 (229.10) (2015) 4172.

[12] K. Farsalinos, K. Poulas, V. Voudris, Changes in puffing topography and nicotine consumption depending on the power setting of electronic cigarettes, Nicotine and Tobacco Research 20 (8) (2018) 993-997

[13] S. Soulet, M. Duquesne, J. Toutain, C. Pairaud, M. Mercury, Impact of vaping regimens on electronic cigarette efficiency, International Journal of Environmental Research and Public Health 16 (23) (2019) 4753

[14] Ecig intelligence: Databases - key global analysis of the vapour sector, https://ecigintelligence.com/content types/database/, retrieved October 28, 2020

[15] M. Manigrasso, G. Buonanno, F. C. Fuoco, L. Stabile, P. Avino, Aerosol deposition doses in the human respiratory tree of electronic cigarette smokers, Environmental Pollution 196 (2015) 257-267.

[16] G. St. Helen, C. Havel, D. A. Dempsey, P. Jacob III, N. L. Benowitz, Nicotine delivery, retention and pharmacokinetics from various electronic cigarettes, Addiction 111 (3) (2016) 535-544.

[17] V. Samburova, C. Bhattarai, M. Strickland, L. Darrow, J. Angermann, Y. Son, A. Khlystov, Aldehydes in exhaled breath during e-cigarette vaping: pilot study results, Toxics 6 (3) (2018) 46.

[18] D. Grégory, E. A. Parmentier, T. Irene, S. Ruth, Tracing the composition of single e-cigarette aerosol droplets in situ by laser-trapping and Raman scattering, Scientific Reports (Nature Publisher Group) 10 (1)

[19] T. Higenbottam, C. Feyeraband, T. Clark, Cigarette smoke inhalation and the acute airway response., Thorax 35 (4) (1980) 246-254.

[20] M. J. Tobin, A. W. Schneider, M. A. Sackner, Breathing pattern during and after smoking cigarettes, Clinical Science 63 (5) (1982) 473-483

[21] D. M. Bernstein, A review of the influence of particle size, puff volume, and inhalation pattern on the deposition of cigarette smoke particles in the respiratory tract, Inhalation toxicology 16 (10) (2004) 675-689.

[22] C. Marian, R. J. O’Connor, M. V. Djordjevic, V. W. Rees, D. K. Hatsukami, P. G. Shields, Reconciling human smoking behavior and machine smoking patterns: implications for understanding smoking behavior and the impact on laboratory studies, Cancer Epidemiology and Prevention Biomarkers 18 (12) (2009) 3305-3320

[23] T. R. Sosnowski, K. Kramek-Romanowska, Predicted deposition of e-cigarette aerosol in the human lungs, Journal of 
aerosol medicine and pulmonary drug delivery 29 (3) (2016) 299-309

[24] C. Xu, P. Nielsen, G. Gong, L. Liu, R. Jensen, Measuring the exhaled breath of a manikin and human subjects, Indoor Air 25 (2) (2015) 188-197.

[25] C. Xu, P. V. Nielsen, L. Liu, R. L. Jensen, G. Gong, Human exhalation characterization with the aid of schlieren imaging technique, Building and Environment 112 (2017) 190-199

[26] C. Y. H. Chao, M. P. Wan, L. Morawska, G. R. Johnson, Z. Ristovski, M. Hargreaves, K. Mengersen, S. Corbett, Y. Li, $X$. Xie, et al., Characterization of expiration air jets and droplet size distributions immediately at the mouth opening, Journal of Aerosol Science 40 (2) (2009) 122-133.

[27] S. Zhu, J.-H. Yang, S. Kato, Investigation into airborne transport characteristics of airflow due to coughing in a stagnant indoor environment., ASHRAE transactions 112 (1).

[28] C. Chen, B. Zhao, Some questions on dispersion of human exhaled droplets in ventilation room: answers from numerical investigation, Indoor Air 20 (2) (2010) 95-111.

[29] J. Wei, Y. Li, Airborne spread of infectious agents in the indoor environment, American journal of infection control 44 (9) (2016) S102-S108.

[30] B. Scharfman, A. Techet, J. Bush, L. Bourouiba, Visualization of sneeze ejecta: steps of fluid fragmentation leading to respiratory droplets, Experiments in Fluids 57 (2) (2016) 24.

[31] J. Askanazi, P. Silverberg, R. Foster, A. Hyman, J. Milic-Emili, J. Kinney, Effects of respiratory apparatus on breathing pattern, Journal of Applied Physiology 48 (4) (1980) 577-580

[32] N. Douglas, D. White, J. Weil, C. Zwillich, Effect of breathing route on ventilation and ventilatory drive, Respiration physiology 51 (2) (1983) 209-218

[33] D. O. Rodenstein, C. Mercenier, D. C. Stanescu, Influence of the respiratory route on the resting breathing pattern in humans, American Review of Respiratory Disease 131 (1) (1985) 163-166

[34] G. H. Yeoh, J. Tu, Computational techniques for multiphase flows, Butterworth-Heinemann, 2019

[35] W. C. Hinds, Aerosol technology: properties, behavior, and measurement of airborne particles, John Wiley \& Sons, 1999 
[36] Z. Ai, C. M. Mak, N. Gao, J. Niu, Tracer gas is a suitable surrogate of exhaled droplet nuclei for studying airborne transmission in the built environment, in: Building Simulation, Springer, 2020, pp. 1-8

[37] E. Ghaem-Maghami, H. Johari, Velocity field of isolated turbulent puffs, Physics of Fluids 22 (11) (2010) 115105.

[38] R. Sangras, O. Kwon, G. Faeth, Self-preserving properties of unsteady round nonbuoyant turbulent starting jets and puffs in still fluids, J. Heat Transfer 124 (3) (2002) 460-469.

[39] R. Sangras, O. Kwon, G. Faeth, Erratum: Self-preserving properties of unsteady round nonbuoyant turbulent starting jets and puffs in still fluids, J. Heat Transfer 125 (3) (2003) 204-205.

[40] G. Buonanno, L. Stabile, L. Morawska, Estimation of airborne viral emission: quanta emission rate of sars-cov-2 for infection risk assessment, Environment International (2020) 105794.

[41] G. Buonanno, L. Morawska, L. Stabile, Quantitative assessment of the risk of airborne transmission of sars-cov-2 infection: prospective and retrospective applications, medRxiv

[42] Propylene glycol used as an excipient report published in support of the 'questions and answers on propylene glycol used as an excipient in medicinal products for human use' (ema/chmp/704195/2013), Available at https://www.ema.europa.eu/en/documents (2017)

[43] B. De Spiegeleer, E. Wattyn, G. Slegers, P. Van der Meeren, K. Vlaminck, L. Van Vooren, The importance of the cosolvent propylene glycol on the antimicrobial preservative efficacy of a pharmaceutical formulation by doe-ruggedness testing, Pharmaceutical development and technology 11 (3) (2006) 275-284

[44] T. T. Puck, The mechanism of aerial disinfection by glycols and other chemical agents: I. demonstration that the germicidal action occurs through the agency of the vapor phase, The Journal of experimental medicine 85 (6) (1947) 729.

[45] T. T. Puck, The mechanism of aerial disinfection by glycols and other chemical agents: li. an analysis of the factors governing the efficiency of chemical disinfection of the air, The Journal of experimental medicine 85 (6) (1947) $741-757$.

[46] T. T. Puck, O. Robertson, H. M. Lemon, The bactericidal action of propylene glycol vapor on microorganisms suspended in air: ii. the influence of various factors on the activity of the vapor, The Journal of experimental medicine 78 (5) (1943) 387-406

[47] V. Vuorinen, M. Aarnio, M. Alava, V. Alopaeus, N. Atanasova, M. Auvinen, N. Balasubramanian, H. Bordbar, P. Erästö, 
R. Grande, et al., Modelling aerosol transport and virus exposure with numerical simulations in relation to sars-cov-2 transmission by inhalation indoors, Safety Science 130 (2020) 104866

[48] Asadi, S., Cappa, C.D., Barreda, S. et al. Efficacy of masks and face coverings in controlling outward aerosol particle emission from expiratory activities. Sci Rep 10, 15665 (2020). https://doi.org/10.1038/s41598-020-72798-7 